TecnoLógicas

ISSN-p 0123-7799

ISSN-e 2256-5337

Vol. 21, No. 41, pp. 29-44

Enero-abril de 2018

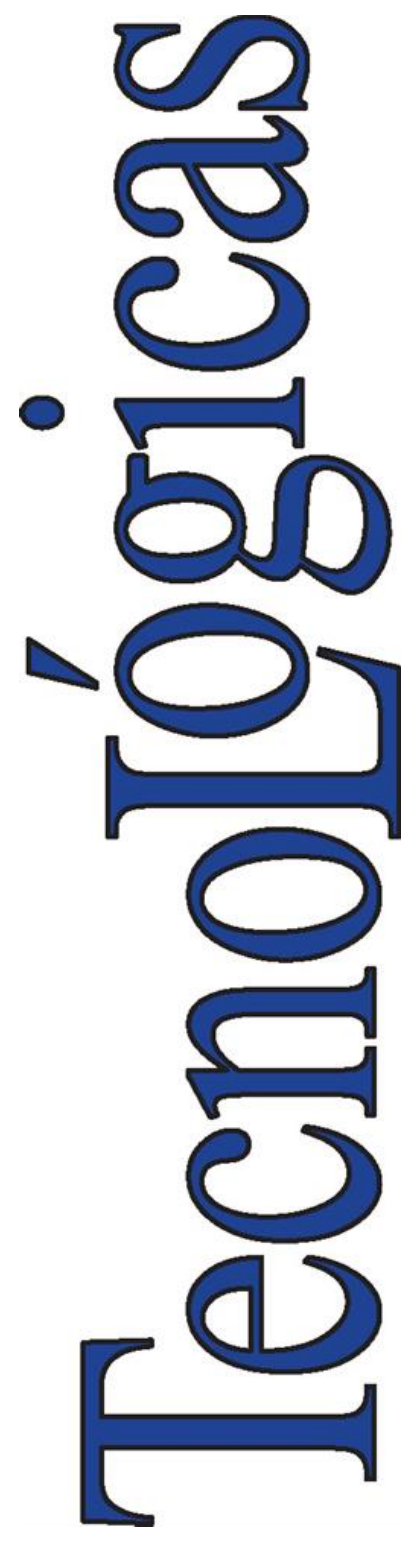

(C) Copyright 2015 por autores y Tecno Lógicas Este trabajo está licenciado bajo una Licencia Internacional Creative Commons Atribución (CC BY)

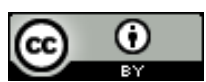

Artículo de Investigación/Research Article

\section{Evaluación del comportamiento térmico de secadores solares en régimen pasivo}

\section{Thermal behavior evaluation of solar dryers in a passive regime}

\author{
Luis E. Mealla-Sánchez¹ y Julián D. Morales-Olaciregui²
}

Recibido: 24 de agosto de 2017

Aceptado: 14 de noviembre de 2017

Cómo citar / How to cite

L. E. Mealla-Sánchez, y J. D. Morales-Olaciregui, Evaluación del comportamiento térmico de secadores solares en régimen pasivo. TecnoLógicas, vol. 21, no. 41, pp. 29-44, 2018.

\footnotetext{
1 MSc. en Energías Renovables, Facultad de Ingeniería, Programa de Ingeniería Mecánica, Departamento de Ciencias Básicas, Universidad Autónoma del Caribe, Barranquilla-Colombia, luis.mealla@uac.edu.co

2 Ingeniero Mecánico, Facultad de Ingeniería, Universidad Autónoma del Caribe, Barranquilla-Colombia, julian.m.ola@gmail.com
} 


\title{
Resumen
}

Se presenta la evaluación del comportamiento térmico de tres secadores solares en régimen pasivo de flujo, diseñados para ser utilizados en las condiciones ambientales de la Costa Caribe Colombiana. Se realizaron experiencias de campo que proporcionaron resultados de eficiencia térmica que se comparan con los proporcionados por una simulación computacional. La simulación se realiza a partir de los balances de energía en cada componente de los secadores. Datos atmosféricos de la locación correspondientes a los días en que se hicieron las pruebas de campo se utilizaron para alimentar la simulación que se realizó usando software específico de licencia de uso libre. Se observa coherencia entre los resultados procedentes de la simulación y los obtenidos a partir de la medición directa.

\section{Palabras clave}

Energía renovable, secador solar, régimen pasivo, eficiencia, simulación computacional.

\begin{abstract}
This article presents the evaluation of the thermal behavior of three solar dryers in a passive flow regime designed for the environmental conditions of the Colombian Caribbean Coast. Field experiments were conducted to obtain thermal efficiency results that were compared with those supplied by a computer simulation. The latter was based on energy balances in each component of the dryers. Atmospheric data of the location on the days the field tests were carried out were used as input for a simulation created using specific opensource software. Consistency is observed between the results from the simulation and those obtained from direct measurement.
\end{abstract}

\section{Keywords}

Renewable energy, solar dryer, passive regime, efficiency, computer simulation. 


\section{INTRODUCCIÓN}

El secado de productos agrícolas para su posterior almacenamiento y conservación resulta primordial cuando no se dispone de energía barata para lograr el cometido. Desde la antigüedad se utiliza esta técnica, así lo demuestra la evidencia proporcionada por instalaciones que datan de 8000 años antes de Cristo [1], consistentes en superficies pavimentadas en donde se depositaba parte de la cosecha para que se secara con radiación directa. Estas superficies frecuentemente se orientan de manera de que la brisa o los vientos predominantes puedan acelerar el proceso de secado. Existe referencia acerca de varios indicios de la utilización de esta técnica para secar telas teñidas y tablillas de arcilla escritas en Mesopotamia alrededor del 7000 al 3000 antes de Cristo. También hacen referencia a la primera evidencia documentada acerca de secado de cosechas en el valle del Indo alrededor del 2600 A.C. Se sabe que el filósofo griego Aristóteles (384 - 322 A.C.) describió el proceso de secado mediante explicación teórica [2]. Este conocimiento artesanal de conservar alimentos aún se puede observar en comunidades rurales en el tiempo presente $[3,4]$.

La necesidad de un flujo de aire caliente, en un recinto aislado, para evitar la contaminación del producto que se deshidrata con polvo, insectos y heces de roedores, es de importancia para asegurar la calidad del mismo. El uso exclusivo de energía solar para producir aire caliente para utilizarlo en la deshidratación de productos necesita además de la provisión de potencia térmica, la remoción de aire mediante una corriente. El uso de bombas o ventiladores garantizan un flujo de aire constante, pero requieren el aporte de una fuente adicional de energía. En este trabajo se propone un balance térmico de tres configuraciones de colectores de aire sometidos a régimen pasivo (sin la intervención de medios externos para la remoción de aire). Se compara su eficiencia térmica, obtenida de pruebas realizadas a prototipos construidos para ese fin, con los resultados obtenidos a través de la simulación computacional a partir de los balances térmicos.

Las ventajas de los secadores solares son muchas, aunque el principal argumento en su contra es el alto costo inicial para su construcción, equipo auxiliar y personal de operación. Se puede enumerar como ventajas: elevada cantidad de energía por unidad de tiempo disponible para evaporación, control en el proceso de deshidratación, no exposición del producto a la intemperie, incremento en la productividad, uso en distintos productos según el uso. No existe un método estándar aceptado mundialmente para la evaluación del comportamiento térmico de los secaderos solares, [5] prepararon un largo listado de propuestas referentes a metodologías de evaluación.

Desde el punto de vista de la eficiencia absoluta del colector, como calentador de aire, [6] propone varios métodos de evaluación conjugando varios estándares internacionales. Se propone adicionar características del secador como costo, tiempo de vida útil, instalación y mantenimiento. Se considera tipo, peso, dimensiones y material con el que se construye el deshidratador. Los más comunes: metal y madera, pero se pueden encontrar alguno hechos de cemento y adobe. Los colectores se construyen de placas de metal (aluminio, hierro galvanizado o acero) cubiertas de pintura no reflectiva negro mate, lámina metálica corrugada y si se requiere temperaturas más elevadas se utilizan materiales selectivos. La cubierta trasparente generalmente es de vidrio o de materiales traslucidos como fluoruro de polivinilo, polipropileno etileno fluorizado, tereheptaleno polipropileno, polietileno, policarbonato, etc. La aislación se hace de lana de vidrio, lana de roca, fibra de coco, aglomerado de aserrín, heno, cáscara de arroz y aserrín. 
León propone tres reglas para mejorar y uniformizar las condiciones constructivas y a su vez evaluar adecuadamente el rendimiento de un secador [5]; estas reglas son adaptadas de [7] de la siguiente manera: (a) Carga promedio del deshidratador (4 kg de producto fresco por metro cuadrado de bandeja). (b) Tamaño del área del colector (0,75 multiplicado por el área total de bandeja). (c) Flujo de aire (0,75 metros cúbicos por minuto por cada metro cuadrado de área de bandeja).

Para evaluar la eficiencia energética de secadores, en la literatura se puede encontrar muchos aportes.

Tiris et al. discuten acerca de la eficiencia térmica de un secadero constituido por un colector y una cámara de secado como una función de los parámetros físicos típicos [8]. Se diseñó el secador para utilizarlo en zonas áridas de Turquía; el calentador de aire de aproximadamente $1,1 \mathrm{~m}^{2}$ de área de colección, compuesto de alambres helicoidales de aluminio pintados de negro como superficie de absorción. Se orientó junto con la cámara de secado hacia el sur y la inclinación del pre-calentador fue de $30^{\circ}$ (la latitud del lugar). Se utilizó poliéster como superficie transparente y el resto fue construido de aluminio y acero inoxidable, se usó lana de vidrio como aislante. $\mathrm{El}$ aire fue impulsado por un ventilador con control manual de velocidad. Se seleccionaron temperaturas de entrada entre 30 y 50 ${ }^{\circ} \mathrm{C}$, los flujos de aire se seleccionaron con valores de 150, 200 y $250 \mathrm{~kg} / \mathrm{m}^{2} \mathrm{~h}$. Se utilizó una resistencia eléctrica para controlar la temperatura de entrada. La radiación solar más baja registrada fue de $630 \mathrm{~W} / \mathrm{m}^{2}$. Los autores definen la eficiencia del colector como el calor útil dividido entre la radiación total que llega al secador. El calor útil se obtiene de la evaluación de la potencia térmica originada en el calentamiento sensible del flujo de aire entre la entrada y la salida del secadero, mientras que la radiación total es el producto de la radia- ción en el plano del secador multiplicado por la superficie trasparente. Presenta los resultados como un ajuste lineal entre la eficiencia instantánea y un parámetro definido como la diferencia entre el promedio de las temperaturas de entrada salida y la temperatura ambiente, todo dividido el calor útil. Se observa pendientes negativas que son más pronunciadas a medida que el flujo de aire disminuye.

En [9], realizaron estudios experimentales para tres distintos tipos de colectores con el fin de decidir cuál de los diseños era óptimo para su uso en secado solar, basados en las normas ASHRAE [10]. La diferencia de su diseño consistía en modificar la superficie de intercambio de calor dentro del colector, utilizaron tres tipos de lámina colectora: plana, aleteada y corrugada con ondas en "v", para distintas condiciones de flujo. Encontraron que este último diseño es el más eficiente. Realizaron pruebas de recirculación de aire sobre las láminas para aumentar la eficiencia. Con esta última metodología se identificó que el más eficiente es el de placa plana, contrario a lo encontrado en las experiencias con un solo paso de aire. Se hicieron pruebas manteniendo cuatro distintos incrementos de temperatura entrada-salida. La irradiación se mantuvo por encima de $790 \mathrm{~W} / \mathrm{m}^{2}$. El cálculo de la eficiencia se realizó haciendo el cociente entre la potencia útil y la potencia total (1). Las integraciones se realizaron en función del periodo de tiempo en el que se trabajó; el calor utilizado para calentar el aire de capacidad calorífica $\mathrm{C}_{\mathrm{p}}$, se calculó en función del flujo de masa y de la diferencia de temperaturas entre la entrada y la salida del colector. La energía total recibida se evaluó en función del área del colector $A_{c}$ y de la irradiación recibida IT. Se trabajó con régimen tendiente a estacionario, aplicando la ecuación de HottelWhillier-Bliss (2) para colectores de aire [11]. 
$\eta=\frac{\dot{m} c_{p} \int_{t_{1}}^{t_{2}}\left(T_{o}-T_{i}\right) d t}{A_{c} \int_{t_{1}}^{t_{2}} I_{T} d t}$

$\eta=\frac{Q_{u}}{A_{c} I_{T}}=F_{R}(\alpha \tau)-F_{R} U_{L} \frac{\left(T_{i}-T_{a}\right)}{I_{T}}$

Se relacionó la eficiencia con los parámetros ópticos radiativos de la cubierta y placa $(\tau \alpha)$, la energía total recibida, el coeficiente global de perdida $U_{L}$, el incremento de temperatura respecto del ambiente. El factor de remoción $F_{R}$ se expresó en función de los demás parámetros.

Maiti et al. [12], realizaron el análisis de la eficiencia de un secadero de convección natural orientado de norte a sur, analizaron el aumento del rendimiento y la eficiencia térmica del colector utilizando reflectores para aumentar la radiación sobre el colector.

Las pruebas tuvieron lugar bajo condiciones de irradiación solar pico durante un día típico en enero en Bhavnagar, Gujarat, India. Realizaron la simulación del secadero para comparar con los resultados de las pruebas obtenidos de un secadero real. El área de colector utilizada fue de $1,8 \mathrm{~m}^{2}$ y la capacidad de cámara fue de $3,46 \mathrm{~kg}$. Los valores medidos dieron un aumento de la eficiencia de colector de 40,0\% a 48,5\% mientras que en la simulación se obtuvieron valores de $36,5 \%$ a 50,3\%. Se probó con un secador de menos área de colector con el que se obtuvo un valor mayor de eficiencia (aproximadamente un $13 \%$ ).

Gabha et al. [13], reportaron pruebas realizadas con un secador con convección natural construido con materiales encontrados en la zona (Costa de Marfil). Presentan estudio de eficiencia en función de los parámetros característicos y el análisis se basa en los datos experimentales mediante un ajuste exponencial empírico. Las pruebas se realizaron en Yamoussoukro, Costa de Marfil, cuya latitud es $9^{\circ}$. El secadero se construyó utilizando una superficie transparente montada similar a un invernadero, en el interior se montó la cámara de secado, y al final se ubicó una chimenea con una placa negra pintada de negro para propiciar la convección natural. Se observó que la eficiencia depende de las temperaturas de entrada y salida, de la rapidez de masa de aire y de la radiación solar incidente.

\section{METODOLOGíA}

Un secador solar, en general, consta de un recinto de secado donde se ubica el material a deshidratar, este puede ser un recinto aislado con un conducto de entrada de aire caliente y un conducto de evacuación de aire húmedo, a menudo se utiliza una cámara o un túnel como recinto para secar. Un sistema de calentamiento de aire, colocado antes del recinto, denominado colector solar, es la principal fuente de energía para el proceso. Básicamente estos dos componentes definen un deshidratador. Adicionalmente, se puede utilizar ductos para conducir el aire, ventiladores, chimeneas, sistemas de control y medida, y mecanismos de acumulación de energía, o en última instancia, fuentes auxiliares para calentar aire o para permitir la circulación de este.

Se optó por construir módulos acoplables para las etapas de colector, cámara de deshidratación y sistema extractor. Se utilizó madera de pino como soporte estructural para las secciones de colector y cámara de deshidratación. Para la superficie de colección del pre-calentador de aire se eligió una lámina de zinc corrugada para tener más superficie de. A partir de allí se acoplaron las diferentes variables de diseño.

La representación de los tres módulos se presenta en la Fig. 1 y se describe de la siguiente manera: el pre-calentador o colector de aire, fue construido con lámina galvanizada ondulada de $0,0002 \mathrm{~m}$ de espesor pintada de negro, de longitud $2,45 \mathrm{~m}$ y ancho $0,81 \mathrm{~m}$. El soporte de colector se 
elaboró con madera de pino de sección cuadrada de 0,0254 $\mathrm{m}$ de ancho, en el interior se cubrió con plástico y el exterior con madera terciada de 0,004 m, se aisló con un espesor de 0,0254 $\mathrm{m}$ de lana de vidrio. La distancia entre la base interna del colector y la cubierta es de $0,1 \mathrm{~m}$, colocando en la zona media la chapa galvanizada apoyada en tres soportes de PVC. La cubierta trasparente se eligió de policarbonato alveolar con superficie anti-UV de 0,01 $\mathrm{m}$ de espesor, con las mismas dimensiones que la lámina galvanizada y enmarcado por perfil de aluminio. Se construyó de tal manera de que al montarse tuviese la inclinación igual a la latitud del lugar.

La cámara de deshidratación, fue construida con una lámina de hierro de 0,0003 $\mathrm{m}$ de espesor, pintada de negro, enmarcada en madera de pino de sección cuadrada de $0.0254 \mathrm{~m}$, cuyas dimensiones interiores son $0,81 \mathrm{~m}$ de ancho y 0,6 de profundidad. La altura que corresponde a la unión con el pre-calentador es de $0,3 \mathrm{~m}$ y la altura de la cara posterior es de $0,42 \mathrm{~m}$, asegurando que el techo de la cámara tenga una inclinación igual que la latitud del lugar. El exterior se cubrió de madera terciada de
0,004 m de espesor y se aisló con lana de vidrio de $0,0254 \mathrm{~m}$. Se dispuso de dos techos desmontables, ambos de 0,81 m por 0,61 m. El primero, se construyó en un marco de madera de pino de sección cuadrada de 0,0254 $\mathrm{m}$ de espesor cubierta en ambas caras con madera terciada de 0,004 $\mathrm{m}$ de espesor y en medio lana de vidrio de 0,0254 m. La segunda cubierta se armó con lámina de policarbonato alveolar anti-UV de 0,01 $\mathrm{m}$ de espesor con la misma superficie que la primera tapa enmarcada en perfil de aluminio.

La chimenea fue construida en lámina de hierro de 0,002 $\mathrm{m}$ de espesor, con un diámetro interior de $0,2 \mathrm{~m}$, apoyada en cuatro soportes de madera de pino, tipo caballete. La chimenea presenta un codo a 0,3 $\mathrm{m}$ a partir de la salida de la cámara de secado y luego una sección vertical de 1,2 $\mathrm{m}$ de altura, encima se colocó un extractor eólico constituido por dos aros metálicos ubicados uno encima de otro que aprisionan un conjunto de alabes paralelos que producen verticidad al paso de viento a través del espacio entre ellos provocando una baja de presión en el interior y posterior extracción de aire.

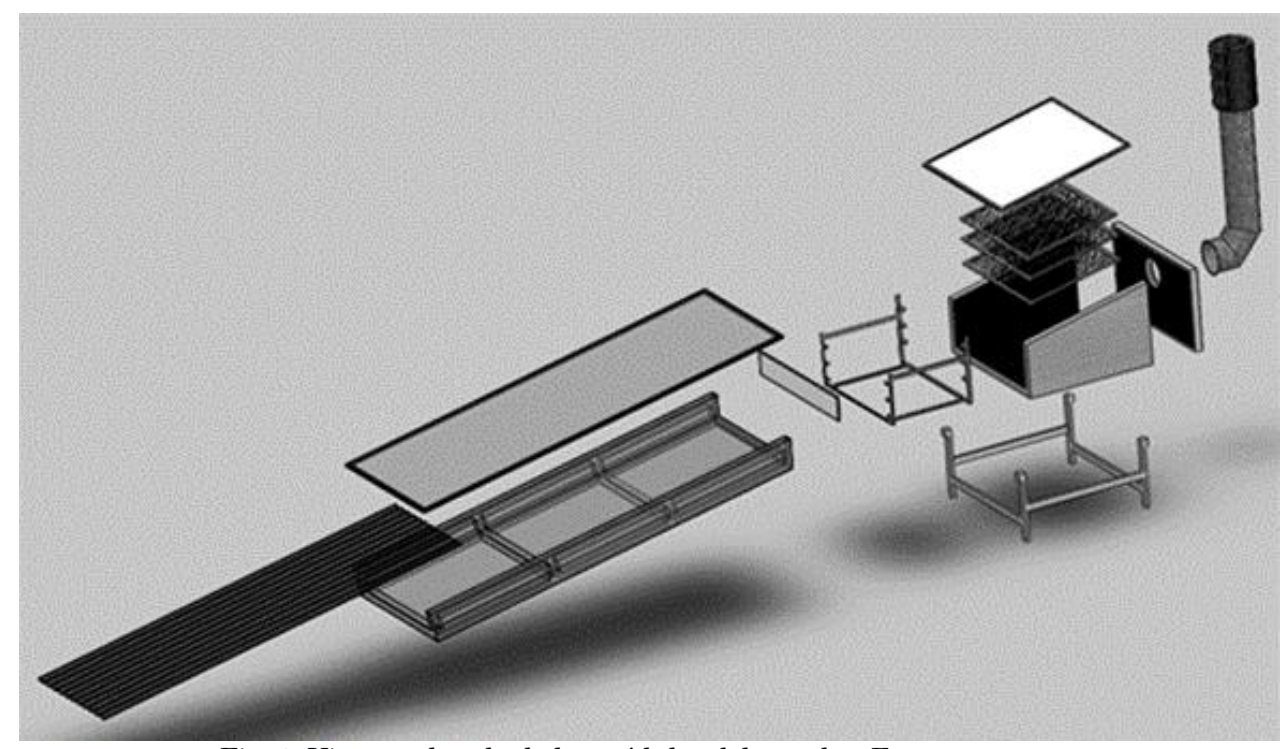

Fig. 1. Vista explotada de los módulos del secador. Fuente: autores. 
El primer prototipo de calentador de aire, denominado mixto, consta de colector, cámara de secado con cubierta trasparente y chimenea. El segundo prototipo, indirecto, consta de colector, cámara de secado con cubierta opaca y chimenea. El último, denominado directo, consta de cámara de secado con cubierta transparente y chimenea.

Se consideran los tres componentes descritos anteriormente para realizar el balance térmico: un colector de aire para precalentar el fluido, la cámara de deshidratación y la chimenea. Se supone que la temperatura es uniforme en cada uno volúmenes de control relacionados con el precalentador y la cámara. En el precalentador se toma siete volúmenes de control con distinta longitud. La cámara de secado se supone con temperatura uniforme en todo volumen centrado y en la chimenea se toma un solo volumen de control, suponiendo que la temperatura del aire no varía apreciablemente en toda su longitud.

El balance térmico para cada uno de los elementos del sistema se realizará siguiendo el esquema análogo de un circuito eléctrico. El aire en el colector utiliza energía para calentarse, la placa colectora acumula energía y solo hay flujo unidireccional. En la cubierta superior del colector, lo ganado por conducción se pierde por radiación a cielo abierto y por convección por acción del viento; no se considera acumulación de energía, como se observa en (3). La ganancia se debe a convección con el aire circulante y radiación desde la placa metálica. La convención de símbolos se muestra en la tabla anexa.
Existe intercambio radiativo entre cubierta y entorno, la temperatura de cielo es $10^{\circ} \mathrm{C}$ menor que la temperatura ambiente. Los coeficientes que representa la pérdida radiactiva dependen de las temperaturas de los objetos que intercambian energía, como se muestra a manera de ejemplo en (4), donde las temperaturas deben expresarse en Kelvin.

El intercambio convectivo depende de la velocidad de viento [14], como se aprecia en (5).

Para calcular los coeficientes de intercambio convectivo en los canales superior e inferior del colector, con el régimen de flujo proporcionado por la chimenea $(\operatorname{Re}<2000$ y $\mathrm{Ra}<10^{7}$ ), los números de Grashof y de Reynolds cumplen con la condición: $0,1<$ $\mathrm{Gr} / \mathrm{Re}^{2}<10$. Los números de Nusselt que se consideran son los correspondientes a flujos naturales y forzados, de los cuales se obtiene un Nusselt combinado; de esas expresiones se puede despejar el coeficiente convectivo $\left(h_{f}=N u \mathrm{k} / \mathrm{D} h\right)$ para los canales del colector [15]. En (6), (7) y (8) el Re se calcula en función del perímetro mojado de cada uno de los canales de flujo. El Ra se calcula con la longitud del colector, que es la más representativa. La ecuación (6) es válida para flujo laminar $\left(\mathrm{Ra}<10^{9}\right)$ (correlación de Churchill y Chu) [16]. La ecuación (7) (correlación de Tan y Chapters) se utiliza para placas paralelas con flujo forzado, una aislada y otra sometida a flujo constante de calor [11], el Pr tiene un valor aproximado de 0,71. Ambas ecuaciones se combinan para tener un Nusselt global [16].

$$
\begin{aligned}
& h_{f c} A_{c}\left(T_{f}-T_{c}\right)+h_{r p-c} A_{c}\left(T_{p}-T_{c}\right)=h_{v} A_{c}\left(T_{c e}-T_{a}\right)+h_{r e c-c l} A_{c}\left(T_{c e}-T_{c l}\left(T_{a}\right)\right) \\
& h_{r e c-c l}=\varepsilon \sigma\left(T_{c}^{2}+T_{c l}^{2}\right)\left(T_{c}+T_{c l}\right) \\
& h_{v}=2,8+3 v
\end{aligned}
$$


$N u_{\text {natural }}=0,68+0,51 R a^{1 / 4}$

$N u_{\text {forzado }}=5,4+\frac{0,00190\left(\operatorname{RePr} D_{h} / L\right)^{1,71}}{1+0,00563\left(\operatorname{RePr} D_{h} / L\right)^{1,17}}$

$N u_{\text {combinado }}=\left(N u_{\text {natural }}^{3}+N u_{\text {forzado }}^{3}\right)^{1 / 3}$

La placa colectora recibe radiación efectiva menor a la radiación solar incidente, ya que es atenuada por la pérdida por transmisión de la cubierta y por su propia absortividad (se supone que la placa acumula energía), esta energía se transfiere por convección hacia el fluido que circula arriba y abajo de ella (calentándolo) y por radiación hacia la cubierta y a la base del colector, como se observa en (9). La base recibe energía por radicación desde la placa y por convección desde el fluido circulante, esta es transmitida por conducción hacia la base y de allí por convección hacia el ambiente.

Los flujos de masa se calculan en función de la velocidad del aire en el sistema, suponiendo continuidad. Se observa en (9) que los flujos de aire arriba y debajo de la placa son complementarios. El coeficiente radiativo entre la placa y la cubierta, la placa y la base, son similares a la ecuación (4), pero intervienen las temperaturas de placa y cubierta en un caso, y las temperaturas de placa y base en el otro caso. Se utiliza en la base el mismo coeficiente convectivo (5).

El balance de energía en la cámara se realiza bajo la suposición de flujo unidireccional y temperatura uniforme en la placa y el aire dentro de la cámara. En la cara externa de la cámara, la cubierta gana energía por conducción a través de ella y se pierde por convección y por radiación; se supone que la cubierta no acumula energía. La cara interior recibe energía en forma radiante de la placa y por convección desde el fluido. La ecuación (10) muestra este balance.
Los coeficientes radiativos se calculan utilizando (4), donde intervienen las temperaturas de cielo, placa y cubierta. El coeficiente convectivo exterior se calcula de la misma manera que en (5). Los coeficientes convectivos en el interior de la cámara se calculan utilizando las correlaciones representadas por (6), (7) y (8). El número de Reynolds se calcula utilizando un diámetro hidráulico promedio y el número de Raleigh se calcula con la longitud característica que es el ancho de la cámara. La energía que recibe la placa se utiliza en calentar la placa y el aire, y en pérdidas convectiva hacia la cubierta y radiativa hacia la base. El flujo másico se supone continuo, por lo tanto, es la suma de los flujos que provienen del colector. Los coeficientes convectivos y radiativos se calculan de forma similar a los que se calculan en el colector. Si se utiliza la configuración indirecta, se debe modificar el balance, debido a que la placa no recibe energía en forma directa. El balance térmico en la chimenea se describe como en (11) y (12).

La cara exterior que recibe radiación total (directa más difusa) pierde una parte por radiación al cielo y convección a la atmosfera, habiendo intercambio de energía radiativo entre ambas semicaras internas. El flujo recibe de ambas semicaras energía por convección, cumple con la condición: coeficiente geométrico H/D mayor que $\mathrm{RaH}^{0,25}$ [17]. El coeficiente convectivo se obtiene a partir de la correlación de Churchill y $\mathrm{Chu}$ ajustada para un $\mathrm{Pr} \cong$ 0,71 [18], expresada en (13).

Despejamos (2), la variación de temperatura de salida respecto de la temperatura de entrada, esto se representa en (14), 
se reemplaza el calor útil por la su definición en un tiempo de integración adecuado. Se utiliza un término auxiliar expresado por (15).

Se puede observar que el salto de temperatura es una función lineal de la potencia radiante recibida por el colector, de manera que la pendiente y el término independiente de un ajuste lineal aparecen como función de las características térmicas y ópticas del colector. Utilizando este criterio se comparan los resultados del factor de remoción producidos por ensayos en tres configuraciones, con los arrojados mediante una simulación computacional, utilizando los balances descritos anteriormente.

Para medir temperatura se construyeron ocho termocuplas tipo " $\mathrm{K}$ " $\left(\mathrm{t} \pm 1,5{ }^{\circ} \mathrm{C}\right)$ conectadas a un sistema de almacenaje de datos Campbell CR1000® (0.18\% del valor recolectado en tensión). Se midió la humedad relativa mediante dos sondas calibradas marca Vaisala ${ }^{\circledR}$ (precisión de $3 \%$ de la medida mostrada en el dispositivo). En el interior del secador se dispusieron dos equipos Lutron ${ }^{\circledR}$ am-4216, y am-4213 (precisión de $\pm 2 \%$ de la medida registrada), uno ubicado en el colector y otro ubicado en la chimenea. La radiación sobre plano inclinado se midió con un sensor de fotodiodo de silicio SP-110 de Apogee Instru- ments ${ }^{\circledR}$ (respuesta espectral de 300 a 1100 $\eta \mathrm{m}, \pm 5 \%$ de la medida). Los datos de temperatura, radiación, velocidad de viento y rapidez en los ductos se tomaron cada cinco minuto, los datos de humedad relativa cada 15 minutos. Se tomaron temperaturas de ambiente, placa de colector, cubierta de colector, entrada a la cámara, chapa de la cámara, producto bandeja inferior, producto bandeja superior, salida de la cámara y chimenea. Adicionalmente, cada sensor de rapidez mide la temperatura en la posición. Cada sensor se protegió de la radiación directa por un cono agujereado de papel de aluminio. La humedad relativa se midió a la salida de la cámara de secado y en el punto donde se ubicó el sensor de temperatura ambiente. La rapidez de aire en el colector y en el ducto de la chimenea se midió en los puntos medios de cada uno de ellos, suponiendo que en esa posición se registran valores máximos de velocidad y son los que se tomaran en cuenta en los cálculos. La velocidad de viento se midió en un punto ubicado a dos metros del prototipo y a dos metros de altura. Se utilizó un anemómetro de cazoleta decagon davis ${ }^{\circledR}$ (precisión $\pm 5 \%$ de la medida registrada). La radiación se midió en plano inclinado. Se realizaron ensayos diarios con cada configuración del prototipo, todas orientadas hacia el sur.

$$
\begin{gathered}
\alpha \tau_{p c} A_{c} I=m_{p} c_{p p} \frac{d T_{p}}{d t}+\dot{m}_{f} c_{p f}\left(T_{i+1}-T_{i}\right)_{f}+h_{f-c}\left(T_{f}-T_{c}\right)+\dot{m}_{f} c_{p f}\left(T_{i+1}-T_{i}\right)_{f} \\
+\left(\frac{A_{b} k_{b} h_{v}}{e_{v} h_{v}+k_{b}}\right)\left(T_{b}-T_{a}\right)+h_{r p-c}\left(T_{p}-T_{c}\right) \\
h_{f c c} A_{c c}\left(T_{f c}-T_{c c}\right)+h_{r p c-c c} A_{c c}\left(T_{p c}-T_{c c}\right)=h_{v} A_{c c}\left(T_{c e c}-T_{a}\right)+h_{r e c c-c l} A_{c c}\left(T_{c c e}-T_{c l}\left(T_{a}\right)\right) \\
\alpha_{t}\left(\frac{A_{t}}{2}\right)\left(I_{d i r}+I_{d i f}\right)=A_{t} h_{r-c}\left(T_{t}-T_{d}\right)+A_{t} h_{v}\left(T_{t}-T_{a}\right)+A_{t} h_{t-f}\left(T_{t}-T_{f}\right) \\
A_{t} h_{t-f}\left(T_{t}-T_{f}\right)+A_{d} h_{d-f}\left(T_{d}-T_{f}\right)=\dot{m}_{f} c_{p f}\left(T_{i+1}-T_{i}\right)_{f}
\end{gathered}
$$


$N u_{H}=\left(0,825+0,328 R a_{H}^{1 / 6}\right)^{2}$

$\left(T_{o}-T_{i}\right)=\frac{A_{c} F_{R}(\alpha \tau) I_{T}}{\dot{m} c_{p}}-\frac{A_{c} F_{R}(\alpha \tau) I_{t h}}{\dot{m} c_{p}}$

$I_{t h}=U_{L} \frac{\left(T_{i}-T_{a}\right)}{\alpha \tau}$

Se realizaron tres simulaciones, una para cada configuración (mixto, indirecto y directo), se utilizó SIMUSOL [19] como programa de cálculo, este usa diagramas de circuitos eléctricos generados por Dia y se resuelven con la ayuda de Sceptre. SIMUSOL interpreta los datos generados y muestra resultados. Se utilizaron características ópticas y térmicas de los materiales que se obtuvieron en tabla. En cada simulación se utilizaron datos de entrada correspondientes a la radiación y la temperatura ambiente registrados en los días de prueba. Se utilizó como variable de control la velocidad del aire en el interior del prototipo. Para que la simulación proporcionara resultados similares a los medidos, se utilizaron promedios de los valores de velocidad registrados.

\section{RESULTADOS Y DISCUSIÓN}

Los resultados de temperatura, humedad relativa, velocidad de ducto, velocidad de viento y radiación se muestran en la Fig. 2; la radiación se compara con un ajuste de Hottel para día claro calculada en plano inclinado. Se muestra como ejemplo los datos tomados para la prueba de calentador mixto, resultados similares se elaboran para las otras dos configuraciones. Se observa que la temperatura de salida de colector en el modo mixto e indirecto presenta una diferencia notable respecto a la temperatura ambiente; en la primera es de aproximadamente $40{ }^{\circ} \mathrm{C}$, mientras que en la configuración en modo indirecto alcanza una diferencia de aproximadamente $35^{\circ} \mathrm{C}$. En el modo directo el salto de temperatura máximo respecto al ambiente es de $20{ }^{\circ} \mathrm{C}$. Se puede observar también que, en los dos primeros modos, la cámara se encuentra prácticamente a la misma temperatura de salida del colector, mientras que en el modo directo llega a una temperatura de $45^{\circ} \mathrm{C}$.

Se observa que temperaturas tienden a comportarse según los cambios de radiación. Los valores de humedad relativa en los tres ensayos arrojan una buena diferencia entre la humedad relativa del ambiente y la alcanzada en el interior de la cámara, lo que proporciona un buen indicio acerca de las condiciones de deshidratación para cualquier producto. La cámara alcanza valores de humedad bajos, acorde a la temperatura que posee. Las medidas de velocidad en el colector y la chimenea, presentan comportamiento proporcional, la chimenea provoca un mayor arrastre dependiendo de la velocidad del viento. El flujo másico en todas las configuraciones es proporcional a ese arrastre reforzado por la fuerza de flotación proporcionada por el calentamiento del aire en el colector en el caso mixto e indirecto, o en la cámara en el caso directo. Se observó presencia de nubes pasajeras durante los días de ensayo. 
Evaluación del comportamiento térmico de secadores solares en régimen pasivo
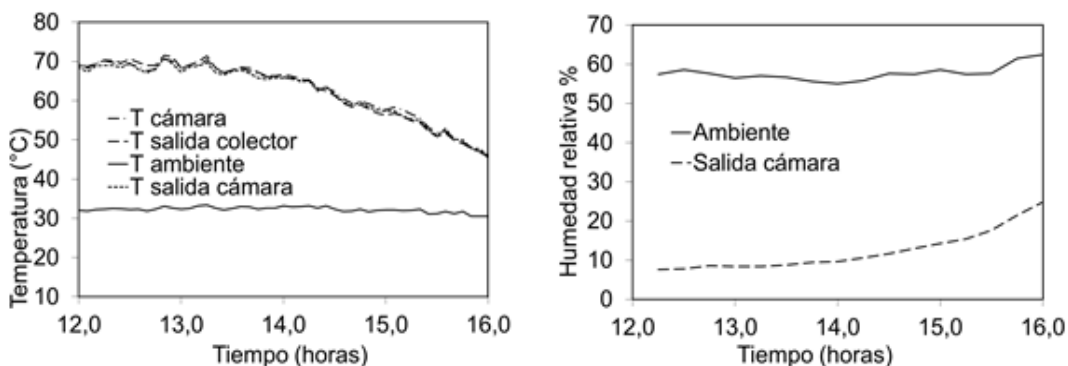

(a)

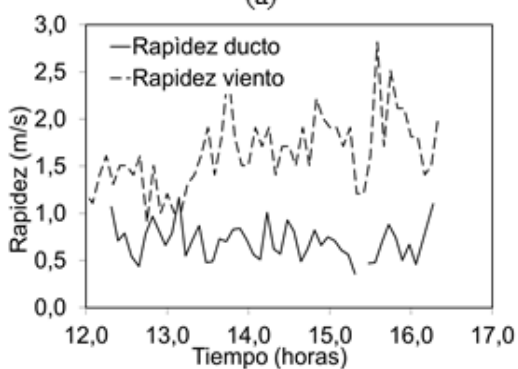

(c)

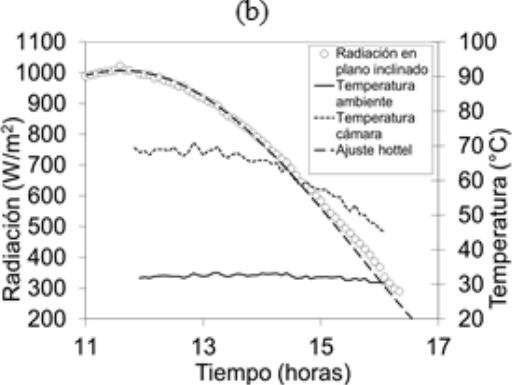

(d)

Fig. 2. Mediciones en la prueba con secador mixto, a) temperatura, b) humedad relativa, c) velocidades de aire, d) radiación en plano inclinado. Fuente: autores.

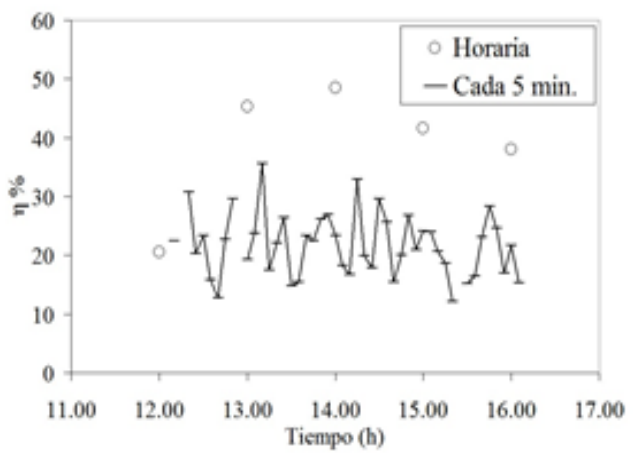

(a)

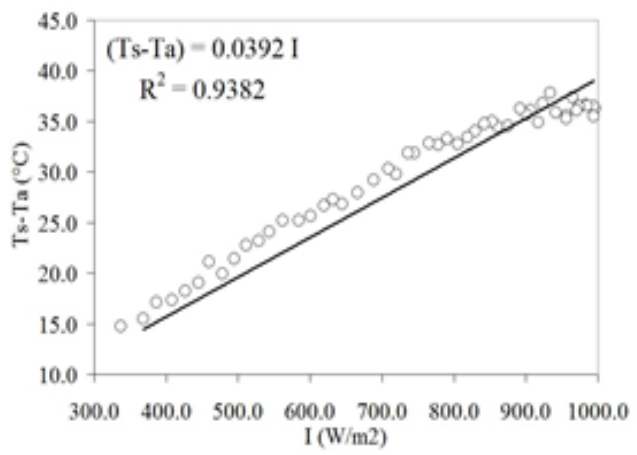

(b)

Fig. 3. Resultados de la prueba térmica para el modo mixto. a) eficiencia en distintos tiempos, b) Salto de temperatura en función de la radiación incidente. Fuente: autores.

La efectividad térmica de las tres configuraciones de calentadores se compara en dos periodos de tiempo, según (1), el cálculo horario comparado con el cálculo cada cinco minutos se muestra en la Fig. 3(a) solo para el modo mixto a manera de ejemplo, generándose dos curvas similares para las otras dos configuraciones. Se calcula el promedio de la eficiencia de la curva proporcionada en el ensayo. La Fig. 3(b) muestra la diferencia de temperatura de salida de la cámara en función de la radiación incidente para la configuración mixto. De allí con los datos de flujo promedio y área de colector, haciendo un ajuste lineal, se pueda obtener el valor del factor de remoción.

La Tabla 1, muestra resultados importantes de los ensayos realizados. Se observa que el modo mixto, presenta un mejor comportamiento térmico en relación con las otras dos configuraciones, esto se debe a la marcada diferencia en el área de colección.

Los resultados provenientes de la simulación se muestran en la Fig. 4, allí se puede comparar las temperaturas del aire caliente de salida con la medida real en la misma posición para tres configuraciones. 
Se observa coherencia entre los resultados del ensayo y el resultado de la simulación. La eficiencia calculada según (1) a través del tiempo, oscila alrededor de un valor promedio debido a las variaciones de la radiación utilizada, pues se tomaron valores de velocidad de aire y viento promedios para calcular el flujo másico y las

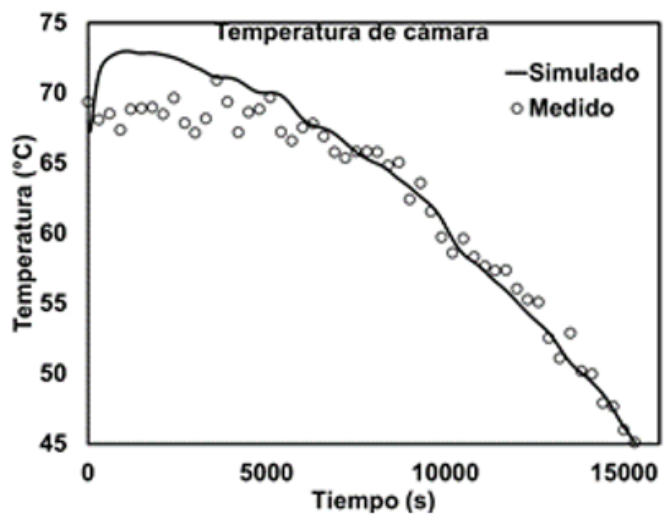

(a) pérdidas convectivas externas. Se analiza la manera en que se desvía la simulación de la medida real, evaluando la desviación estándar, el error estándar y el coeficiente de correlación según lo sugerido por la literatura [20]. Los valores obtenidos se muestran en la Tabla 2.

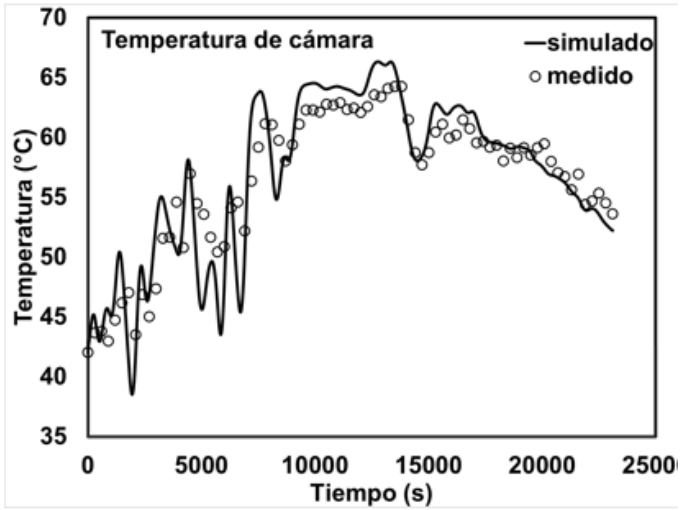

(b)

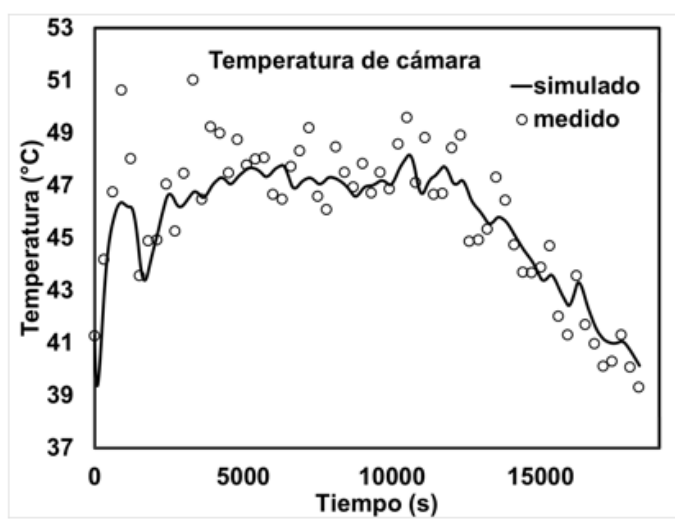

(c)

Fig. 4. Temperaturas de salida. Simuladas y medidas. a) mixto. b) indirecto. c) directo. Fuente: autores.

Tabla 1. Resultados del análisis de los datos proporcionados por las pruebas de campo. Fuente: autores.

\begin{tabular}{cccccc}
\hline $\begin{array}{c}\text { Tipo de } \\
\text { secador }\end{array}$ & $\begin{array}{c}\text { Flujo promedio } \\
(\mathrm{kg} / \mathrm{s} \mathrm{m} 2)\end{array}$ & $\begin{array}{c}\text { Área de colección } \\
(\mathrm{m} 2)\end{array}$ & $\begin{array}{c}\text { Pendiente }\left({ }^{\circ} \mathrm{C} / \mathrm{W} / \mathrm{m} 2\right) \\
\frac{A_{c} F_{R}(\alpha \tau)}{\dot{m} c_{p}}\end{array}$ & $\begin{array}{c}\text { Eficiencia promedio } \\
\text { en porcentaje }\end{array}$ & $\begin{array}{c}F_{R} \\
\text { promedio }\end{array}$ \\
\hline Mixto & 0,29 & 2,5 & 0,039 & 22 & 0,778 \\
$\begin{array}{c}\text { Indirec- } \\
\text { to }\end{array}$ & 0,17 & 2,0 & 0,031 & 18 & 0,453 \\
Directo & 0,05 & 0,5 & 0,015 & 1,2 & 0,258 \\
\hline
\end{tabular}

Tabla 2. Resultados estadísticos de la comparación entre medidas reales y simuladas para la temperatura de cámara. Fuente: autores.

\begin{tabular}{cccc}
\hline secador & desviación estándar & error estándar & coeficiente de correlación \\
\hline Mixto & 2,158 & 0,302 & 0,981 \\
Indirecto & 2,563 & 0,292 & 0,995 \\
Directo & 1,297 & 0,166 & 0,905 \\
\hline
\end{tabular}


Se observa que la desviación en temperatura en la cámara, desde el punto de vista estadístico, no es muy grande teniendo en cuenta la fluctuación de la radiación solar en los días de prueba. Estos valores confirman que los datos proporcionados por la simulación ajustan de manera adecuada a los valores reales. Esto lo corrobora el bajo valor del error estándar y la cercanía a uno del error de correlación.

Se realizó el cálculo de la eficiencia instantánea comparando el calor necesario para calentar una determinada masa de aire con la potencia radiante por unidad de área que llega a la cubierta transparente de cada uno de los prototipos ensayados, tal como lo indica en (2). Utilizando los valores producidos por la simulación se obtuvo la eficiencia instantánea que se muestran en la Fig. 5.

Las variaciones bruscas se deben a la variación en la intensidad de la radiación registrada en el plano de la superficie transparente. Haciendo el cálculo promedio, se observa coherencia en cuanto a la tendencia que muestran los resultados reales, la configuración mixto muestra un valor promedio de 44,3\%, la configuración indirecto $32,5 \%$ y la configuración directo $25,5 \%$.

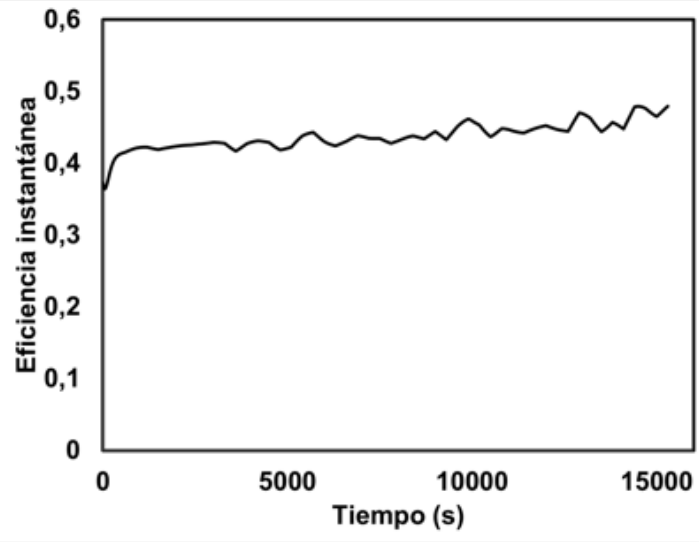

(a)

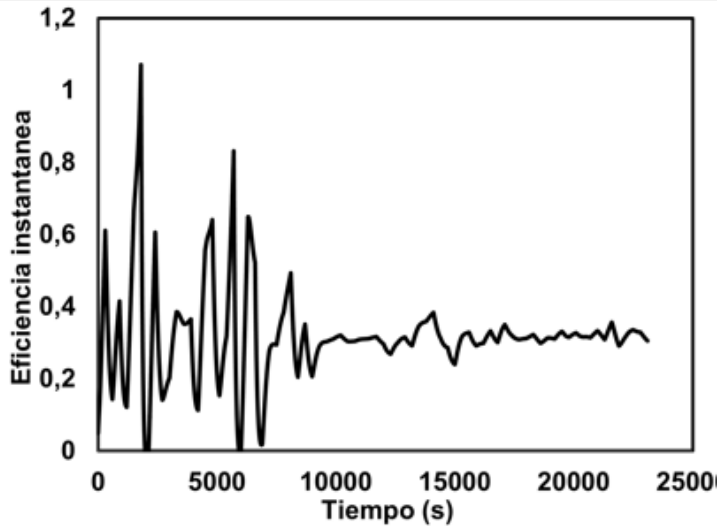

(b)

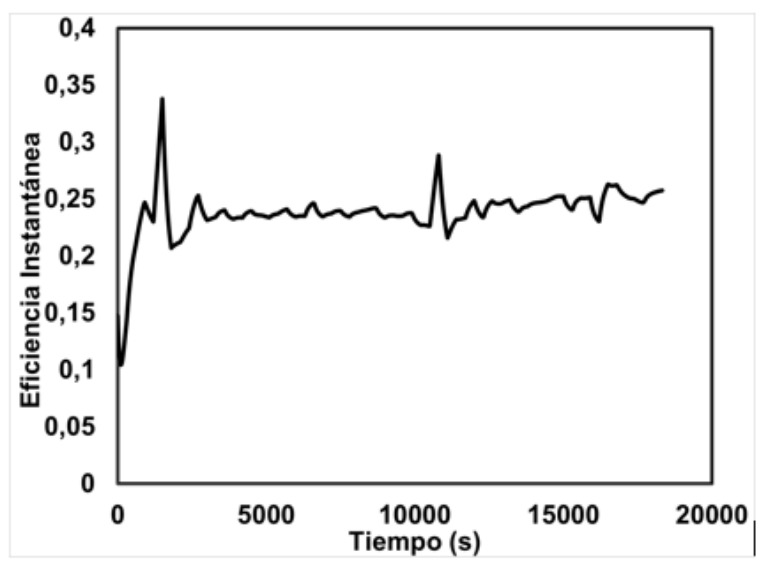

(c)

Fig. 5. Eficiencia instantánea. a) mixto. b) indirecto. c) directo. Fuente: autores. 


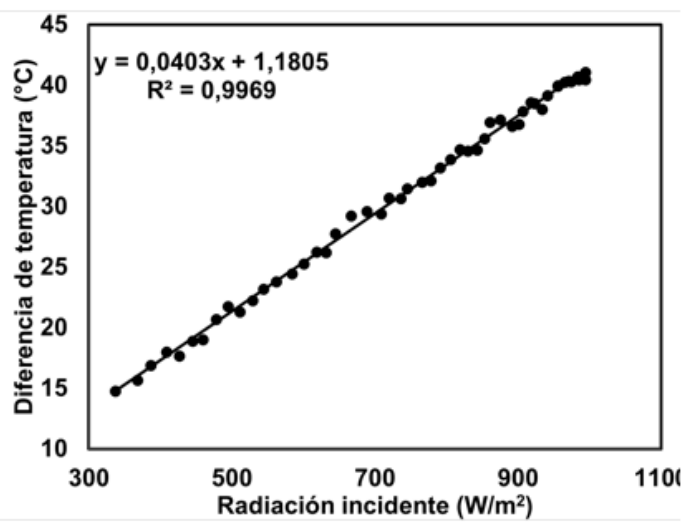

(a)

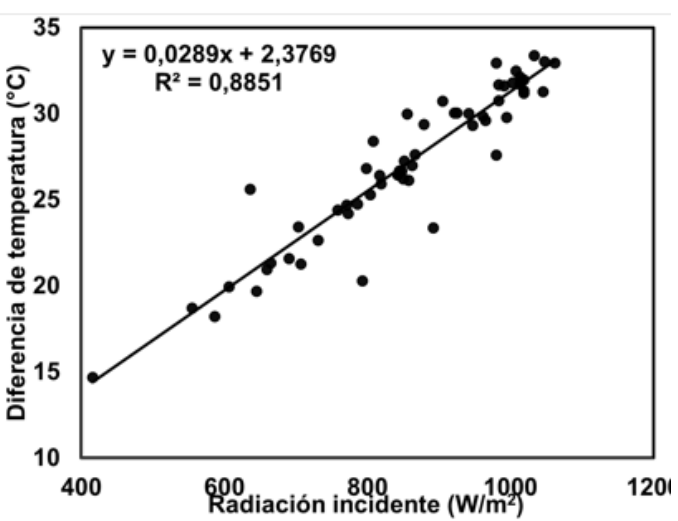

(b)

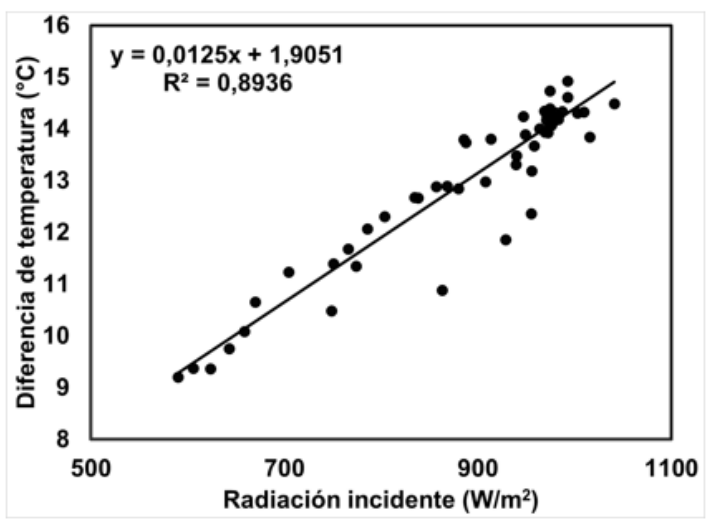

(c)

Fig. 6. Diferencia de temperatura entre la cámara y el ambiente en función de la radiación incidente. a) mixto. b) indirecto. c) directo. Fuente: autores.

Se calculó la diferencia de temperatura entre la cámara y el ambiente. Estos valores proceden de la simulación. Se tomaron solamente los valores correspondientes a los tiempos que tienen las tablas que alimentan cada una de las simulaciones, de esta manera se eliminó mucha información espuria y se obtuvieron resultados similares a los calculados con los datos medidos. El salto de temperatura se graficó en función de la radiación incidente que procede de la simulación. La Fig. 6 presenta esos resultados.

Se puede observar que el ensayo con el secador en modo mixto, produce un ajuste lineal más significativo comparado con los ensayos en las otras dos configuraciones. Esto se debe a las condiciones de variación de la radiación solar en los días de ensayos.

Utilizando la información proporcionada por el ajuste lineal entre la diferencia de temperatura y la radiación solar incidente, se calculó el factor de remoción para cada uno de los prototipos, teniendo en cuenta los valores de flujo promedio para cada corrida de datos y las constantes utilizadas en cada simulación que aparecen en la Tabla 3. Se utilizó una relación transmitancia-absortancia de 0,64 y una densidad de aire promedio de $1,09 \mathrm{~kg} / \mathrm{m}^{3}$.

Se observa que existe una marcada diferencia en el valor de la pendiente, pues el área que colecta radiación es la que determina la potencia que llega a cada una de las configuraciones de secadero. El factor de remoción es un resultado de las condiciones de simulación. Se observa que la tendencia es similar a lo ocurrido cuando se realiza el mismo cálculo con los valores medidos. 
Evaluación del comportamiento térmico de secadores solares en régimen pasivo

\begin{tabular}{cccccc}
\multicolumn{5}{c}{ Tabla 3. Resultados de la simulación. Fuente: autores. } \\
\hline Tipo de secador & $\begin{array}{c}\text { Flujo } \\
\text { promedio } \\
(\mathrm{kg} / \mathrm{s} \mathrm{m} 2)\end{array}$ & $\begin{array}{c}\text { Área de } \\
\text { colección } \\
(\mathrm{m} 2)\end{array}$ & $\begin{array}{c}\text { Pendiente }\left({ }^{\circ} \mathrm{C} / \mathrm{W} / \mathrm{m} 2\right) \\
\frac{A_{c} F_{R}(\alpha \tau)}{\dot{m} c_{p}}\end{array}$ & $\begin{array}{c}\text { Eficiencia promedio } \\
\text { en porcentaje }\end{array}$ & $\begin{array}{c}F_{R} \\
\text { promedio }\end{array}$ \\
\hline Mixto & 0,25 & 2,5 & 0,0401 & 44,3 & 0,630 \\
Indirecto & 0,16 & 2,0 & 0,0231 & 32,5 & 0,390 \\
Directo & 0,09 & 0,5 & 0,0102 & 25,5 & 0,288 \\
\hline
\end{tabular}

\section{CONCLUSIONES}

Se ha presentado la comparación del comportamiento térmico de tres configuraciones de secadores solares con régimen de extracción natural de aire. La validación de las pruebas de campo se realizó a través de una simulación computacional que tuvo en cuenta los datos medidos en distintos ensayos térmicos realizados con los prototipos. Los balances de energía toman en cuenta la existencia de dos canales en un colector, destinado para calentar aire en un flujo circulante que es causado por una chimenea con un extractor que genera verticidad por viento. Se consideran los intercambios de calor dentro del colector, de tipo convectivo y radiativo, y las pérdidas de calor por la cubierta al ambiente, de tipo convectivas y radiativas, y a través del cuerpo del colector hacia el ambiente, de tipo conductivas en el cuerpo del colector, y convectivas en el exterior. En la cámara se realizó la misma suposición, pero con un solo flujo de aire. La chimenea producía el flujo por flotación, sumada a la variación de la densidad del aire en todo el sistema.

Se realizó una simulación computacional del colector mediante el uso de Simusol, considerando las hipótesis detalladas. Esta simulación se llevó a cabo tomando como entrada valores de temperatura ambiente, flujo de aire y radiación solar; contrastando los resultados medidos y simulados de temperatura de aire a la salida de la cámara de secado, la eficiencia como colector y el factor de remoción como indicador de la eficiencia promedio de cada prototipo. La comparación se realizó entre los resultados simulados y los medidos, observándose un buen ajuste entre resultados medidos y simulados, tanto en temperaturas de flujo de aire, las cuales muestran estadísticamente los parámetros adecuados. Los factores de remoción y las curvas de eficiencia presentan comportamientos similares. Queda como inquietud, el realizar el mismo protocolo de pruebas para las tres configuraciones, utilizando flujo forzado y un sistema de retroalimentación de aire, para poder comprar este tipo de comportamiento térmico con los que muestra el presente trabajo.

\section{REFERENCIAS}

[1] V. Belessiotis and E. Delyannis, "Solar drying," Sol. Energy, vol. 85, no. 8, pp. 16651691, Aug. 2011.

[2] K. Kröll and W. Kast, Trocknen und Trockner in der Produktion. Berlin, 1989.

[3] O. V Ekechukwu and B. Norton, "Review of solar-energy drying systems III: low temperature air-heating solar collectors for crop drying applications," Energy Convers. Manag., vol. 40, no. 6, pp. 657-667, 1999.

[4] G. Duran, M. Condori, R. Echazú, and G. D. Russo, "Secador solar híbrido para la producción continúa a escala industrial de pimiento para pimentón," in IV Conferencia LatinoAmericana de Energía Solar (IV ISES_CLA), 2010, pp. 1-15.

[5] Ma. Leon, S.C Bhattacharya, and S. Kumar, "A comprehensive procedure for performance evaluation of solar food dryers," Renew. Sustain. Energy Rev., vol. 6, no. 4, pp. 367393, Aug. 2002.

[6] M. S. Sodha and R. Chandra, "Solar drying systems and their testing procedures: A review," Energy Convers. Manag., vol. 35, no. 3, pp. 219-267, Mar. 1994.

[7] G. M. Tokar, "Food drying in Bangladesh," Dhaka: Agro-based industries and technology Project (ATDP), 1997.

[8] C. Tiris, M. Tiris, and I. Dincer, "Investigation of the thermal efficiencies of a solar dryer," Energy Convers. Manag., vol. 36, no. 3, pp. 205-212, Mar. 1995.

[9] M. A. Karim and M. N. A. Hawlader, 
"Development of solar air collectors for drying applications," Energy Convers. Manag., vol. 45, no. 3, pp. 329-344, Feb. 2004.

[10] R. American Society of Heating and AirConditioning Engineers, Methods of testing to determine thermal performance of solar collectors. New York: ASHRAE, 1978.

[11] J. A. Duffie, W. A. Beckman, and W. M. Worek, "Solar Engineering of Thermal Processes," J. Sol. Energy Eng., vol. 116, no. 1, p. 67, 1994.

[12] S. Maiti, P. Patel, K. Vyas, K. Eswaran, and P. K. Ghosh, "Performance evaluation of a small scale indirect solar dryer with static reflectors during non-summer months in the Saurashtra region of western India," Sol. Energy, vol. 85, no. 11, pp. 2686-2696, Nov. 2011.

[13] P. Gbaha, H. Yobouet Andoh, J. Kouassi Saraka, B. Kaménan Koua, and S. Touré, "Experimental investigation of a solar dryer with natural convective heat flow," Renew. Energy, vol. 32, no. 11, pp. 1817-1829, Sep. 2007.
[14] W. H. McAdams, "Heat Transmission", Mcgraw, 3rd ed., no. 4. 1954.

[15] G. Duran, M. Condorí, and F. Altobelli, "Simulation of a passive solar dryer to charqui production using temperature and pressure networks," Sol. Energy, vol. 119, pp. 310-318, 2015.

[16] [16] F. P. Incropera, D. P. DeWitt, T. L. Bergman, and A. S. Lavine, Fundamentals of Heat and Mass Transfer, 6th ed. John Wiley \& Sons, Inc., 2007.

[17] M. Condorí, L. Mealla, and L. Saravia, "Estudio y modelización de un nuevo diseño de chimenea solar," Av. en Energía Renov. $y$ Medio Ambient., vol. 5, pp. 19-24, 2001.

[18] A. Bejan, Convection Heat Transfer. Hoboken, NJ, USA: John Wiley \& Sons, Inc., 2013.

[19] D. Alia, L. R. Saravia, and D. Saravia, "Simusol: simulating thermal systems using sceptre and DIA," J. Free Softw. Free Knowl., vol. 1, pp. 30-34, 2012.

[20] J.P. Holman, Experimental Methods for Engineers, 8th ed. New York: McGraw-Hill Education, 2011. 